\title{
Direct and iterative computing of fluid flows fully coupled with structures
}

\author{
Hou Zhang ${ }^{\mathrm{a}, *}$, Klaus-Jürgen Bathe ${ }^{\mathrm{b}}$ \\ ${ }^{a}$ ADINA R\&D, Inc., 71 Elton Avenue, Watertown, MA 02472, USA \\ ${ }^{b}$ Massachusetts Institute of Technology, Mechanical Engineering Department, Cambridge, MA 02139, USA
}

\begin{abstract}
The ADINA program can be used for analyses of fluid flows fully coupled with structures. Fluid flows can be modeled as incompressible, slightly compressible, fully compressible and porous media flows. Both iterative (partitioned) and direct (simultaneous) solution procedures can be used. The capabilities available in ADINA are briefly summarized and various experiences regarding the use of the two solution procedures are given. It is concluded that it is valuable to have both analysis approaches in one code.
\end{abstract}

Keywords: Fluid-structure interaction; Simultaneous procedure; Partitioned procedure

\section{Introduction}

The analysis of fluid-structure interaction (FSI) problems, in which the fluid is modeled using the general Navier-Stokes or Euler equations, has been given increased attention in the recent years. The reason is that the numerical methods and the computer hardware have now reached the capacities that FSI problems can be realistically modeled and solved in engineering and scientific analyses. In various fields of scientific investigations, the fluid flows fully coupled with structural deformations represents the essence of the problems considered, for example in biomechanical analyses; and in many engineering designs the interaction between the fluid flows and structures needs to be investigated in order to reach safe and economical, and therefore competitive designs.

Various approaches have been followed to obtain an FSI solution capability. With an efficient fluid solver available, simple structural elements have been programmed into the solver (such as spring elements) and certain FSI problems have been solved efficiently. Another more common approach is to couple fluid and structural solvers by data transfer between the solvers; the data is transferred so as to satisfy, by iteration, in each incremental solution step

\footnotetext{
* Corresponding author. Tel.: +1 (617) 926-5199; Fax: +1 (617) 926-0238; E-mail: hou@adina.com
}

the physical conditions on the fluid-structure interfaces. This approach is commonly referred to as the 'partitioned' or 'iterative' solution procedure. Sometimes, the iteration is not performed and the solution is simply attempted by passing data at the beginning/end of the incremental steps from one to the other solver.

The most powerful solution approach appears to be to 'simply' set up the fully coupled governing fluid flow and structural equations and solve the equations using the Newton-Raphson method, all in one code. This approach is referred to as the 'simultaneous' or 'direct' solution procedure.

The objective of this paper is to briefly summarize the partitioned (iterative) and simultaneous (direct) solution procedures available in ADINA, and to present some experiences with the approaches. The fact that both solution approaches are available in one code renders it possible to make direct comparisons between the approaches - of course, as implemented in ADINA.

\section{ADINA for FSI solutions}

The ADINA program is a general-purpose finite element/finite volume code for the analysis of structures, fluids and FSI problems [1]. Structures are modeled using finite element procedures. The structural response can 
be linear or highly nonlinear, including contact conditions. A general Lagrangian formulation for the structural response is used. Fluids are modeled using the general Navier-Stokes equations (or Euler equations). The usual Eulerian formulation is solved when the fluid boundaries do not move, i.e. when there is no prescribed wall movement and there are no structural interactions. An arbitrary Lagrangian-Eulerian (ALE) formulation is employed when there is movement of the fluid boundaries. The NavierStokes equations of incompressible flows are solved using finite element procedures (mixed velocity/pressure based discretizations are used), whereas the Navier-Stokes equations of high-speed compressible flows are solved using control volume procedures. For slightly compressible and low-speed compressible flow solutions, a mixed finite element/finite volume discretization is employed. In each case, high Reynolds and Peclet number flows require the use of an upwinding procedure that has been derived from control volume techniques [1,2].

An acoustic fluid assumption can also be used, in which case the fluid response is calculated by a potential formulation. Then the fluid finite element discretization results into symmetric coefficient matrices that are coupled to the structural stiffness matrices. Hence, in this case, the simultaneous solution approach is always employed. We do not consider this formulation further in this paper, but refer to $[1,2]$.

The key in the coupling of the Navier-Stokes or Euler fluid flow discretized equations to the structural discretized equations lies in how the interface conditions are imposed. At fluid structural interfaces, the conditions of compatibility of displacements/velocities and of stress equilibrium must be fulfilled. The relevant equations on the interface are:

displacement compatibility,

$\mathbf{d}_{f}^{S}=\mathbf{d}_{s}^{S}$

where $\mathbf{d}_{f}^{S}$ and $\mathbf{d}_{s}^{S}$ are the displacements of the fluid and solid, respectively; and

traction equilibrium,

$\mathbf{f}_{f}^{S}=\mathbf{f}_{s}^{S}$

where $\mathbf{f}_{f}^{S}$ and $\mathbf{f}_{s}^{S}$ are the tractions of the fluid and solid on the interface, respectively.

Consider a general structural mesh (using 2D, 3D, beam or shell elements) and a general fluid mesh, using of course entirely different elements, in size and otherwise. Usually, a much finer fluid mesh than structural mesh is needed. The conditions of compatibility then require that the fluid mesh 'abut' to the structural mesh using the displacement interpolations of the structural elements. Of course, the fluid mesh can slide on the structural mesh in the ALE formulation of fluid flow. If the Navier-Stokes equations are solved for the fluid, then, at the interface, the velocities of the fluid particles must be equal to the velocities of the structural particles (no slip condition), whereas if the Euler equations are used to model the fluid, the fluid particles are free to slip along the interface, that is, along the structural mesh (of course at the physical relative velocity, which is in general different from the fluid mesh velocity). In ADINA, these conditions are imposed using the different element interpolation functions for displacements (and velocities).

The conditions of stress equilibrium require that the stresses in the fluid be balanced by the stresses in the structure on the interface. The forces exerted onto a structural element are

$\mathbf{F}(t)=\int \mathbf{H}^{S^{\mathrm{T}}} \mathbf{f}_{f}^{S} \mathrm{~d} S$

where the matrix $\mathbf{H}^{S}$ stores the shape functions of the element and the vector $\mathbf{f}_{f}^{S}$ contains the tractions exerted by the fluid. Note that in this equation, the nodal consistent forces are evaluated, which is an important requirement for an accurate analysis (the patch condition is satisfied [2]).

In ADINA, the iterative solution procedure solves the fluid flow equations for the last calculated structural configuration, then calculates the forces in Eq. (3), applies these forces onto the structural model, evaluates incremental structural displacements, applies these incremental structural displacements onto the fluid model, and continues to iterate until convergence for that load/time step is reached. The iteration is effective because the structural and fluid flow solvers are called as subroutines, using dynamically allocated memory, with all shared data directly available to both solvers. Hence the amount of disk reading and writing is minimized. Since the fluid flow and structural equations are solved sequentially, the same memory is used for the structural and the fluid flow coefficient matrices. Of course, at convergence the fully coupled solution has been obtained.

If, in this solution approach, no iteration is performed, but the solution is simply marched forward, then for somewhat strongly coupled problems, errors in the solution accumulate and may well lead to instability of the response prediction. This phenomenon is quite similar to what is seen if no iteration is performed in a nonlinear dynamic analysis (see [2]). Hence, it is best to always perform iterations to reach convergence to within reasonable convergence tolerances.

The direct solution procedure in ADINA solves the same governing equations for the structure and the fluid as the partitioned procedure, but obtains the solution by solving these equations simultaneously using one coefficient matrix. This matrix contains the same structural and fluid matrices as in the partitioned procedure plus a Jacobian matrix corresponding to the interface conditions, obtained by imposing directly the compatibility conditions and by linearization of Eq. (3). 


\section{A sample solution}

Both approaches have been applied to the solutions of many problems. Here, we present one simple but demonstrative solution.

Fig. 1 shows the problem considered. A sudden prescribed displacement onto the piston causes a pressure wave in the fluid, which moves the steel ball vertically up to the top of the chamber. Fig. 2 shows various configurations of the steel ball during its travel to the top of the chamber. The difficulties in the solution result from the large travel of the ball and the contact/gap conditions for the ball in the initial configuration and when the ball hits the top of the chamber. Fig. 3 shows the vertical displacements of the ball and the piston as functions of time. The same solution was obtained by the direct and iterative

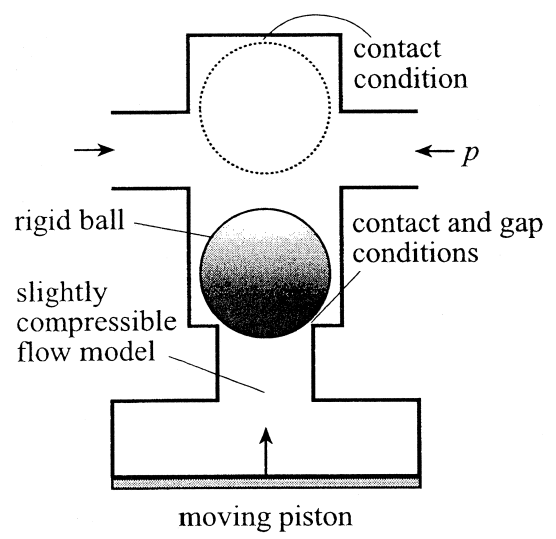

Fig. 1. Example problem (ratios of dimensions physically as shown in figure). procedures, although the most effective way is to use the partitioned approach when the contact/gap conditions are active and the direct procedure at the other solution steps.

\section{Conclusions}

Considering the iterative and direct solution procedures in ADINA, the following conclusions are reached.

- The direct procedure requires considerably more storage, but can be used to solve problems for which the iterative procedure cannot be used due to the large nonlinearities present.

- The iterative procedure is usually effective if convergence in the iterations is achieved using a reasonable number of iterations per step and the number of solution steps does not need to be much larger than when using the direct procedure.

- The iterative procedure becomes more effective as the size of the model considered increases.

- If the structural model contains only a few degrees of freedom (in comparison with the fluid model), the direct procedure is frequently the most effective method.

Of course, the most effective way to proceed is to use an adaptive procedure, which automatically chooses during the solution path, at the solution steps, the direct or iterative procedure depending on which approach is more effective. An example of such solution was given above.

\section{References}

[1] ADINA R\&D, Inc., ADINA Theory and Modeling Guide, Volumes I, II and III, Watertown, MA, 2000.

\section{ADINA}
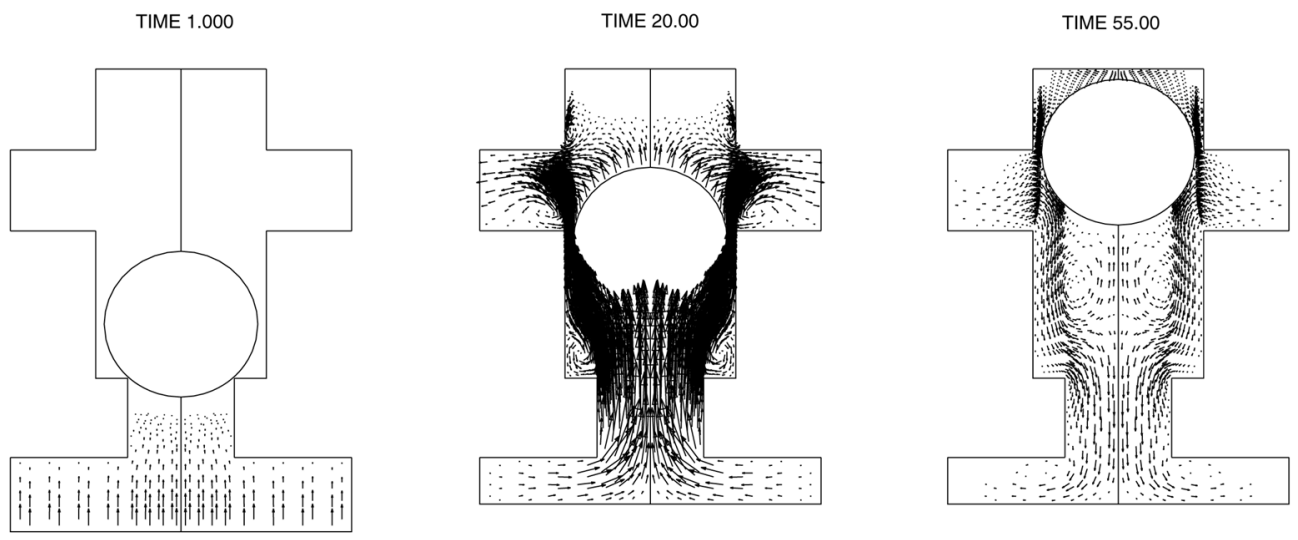

Fig. 2. Various configurations of the steel ball and velocity fields. 


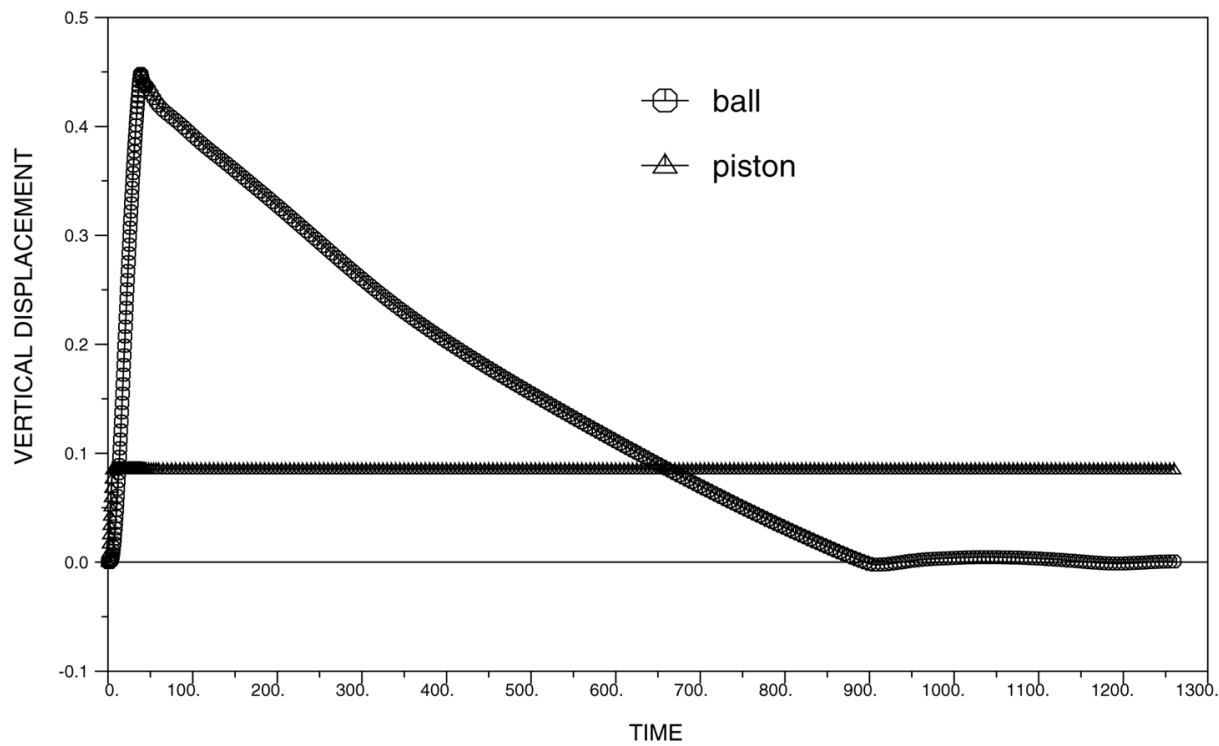

Fig. 3. Vertical displacements of the steel ball and the piston.

[2] Bathe KJ. Finite Element Procedures. Prentice Hall, Englewood Cliffs, NJ, 1996.
[3] Bathe KJ, Zhang H, Ji S. Finite element analysis of fluid flows fully coupled with structural interactions, Comput Struct 1999;72:1-16. 